\title{
Connected Media and Presence
}

\author{
Joke Kort \\ TNO \\ Eemsgolaan 3 \\ 9727 DW Groningen \\ The Netherlands \\ +31 (0)888 667751 \\ joke.kort@tno.nl \\ Harold Nefs \\ Delft University of Technology \\ Department of Electrical Engineering, Mathematics, and Computer Science \\ Mekelweg 4, 2628 CD Delft, \\ The Netherlands \\ h.t.nefs@tudelft.nl \\ Charlie Gullström \\ KTH Royal Institute of Technology, Dept. Architecture \\ 10044 Stockholm \\ Sweden \\ +46 (8)7 909211 \\ charlieg@kth.se \\ Tjerk de Greef \\ Delft University of Technology \\ Department of Electrical Engineering, \\ Mathematics, and Computer Science \\ Mekelweg 4, 2628 CD Delft, \\ The Netherlands \\ t.e.deGreef@tudelft.nl \\ Peter Parnes \\ Lulea University of Technology \\ Department of Computer Science, \\ Electrical and Space Engineering \\ 97187 Luleå \\ Sweden \\ Peter.Parnes@|tu.se
}

\begin{abstract}
Effective design of shared mediated spaces, information and connectedness requires theory and practice from a range of disciplines such as found in European projects like Together Anywhere, Together Anytime (TA2) and the EIT ICT Labs Mediating Presence activity. Building on this work we continue to investigate the changes in the European digital media industry such as changed traditional distribution of media content and the progressive integration of (social) communication means in information distribution and shared mediated spaces. Our past research has given valuable insights in how to design and evaluate systems and services that provide a high quality of experience, in how trust is established in mediated environments, and how the formation of tacit communication between participants in new distributed and
\end{abstract}


connected media is negotiated. In the new Seventh Framework Program project COnnected Media and Presence from European Institute of Technology (COMPEIT) we aim to enhance the quality of experience in face-to-face and broadcast communication further in three domains: 1) Spatial connectedness, 2) Social connectedness and 3) Information connectedness, by developing three key services: Shared Experience with Tangible Interaction (SETI); Broadcast Presence Studio (BPS) and Mixed-Reality Interaction (MRI). The quality of experience of these services will be enhanced in terms of for example: spatial connectedness, by providing shared spaces and supporting spatial features such as eye contact; social connectedness, by using natural means for interaction suiting different settings and activities; and information connectedness, by providing better means to share, manipulate and use information suiting different task or activities. In this paper we will discuss the background of this work and give an overview of our planned future work in COMPEIT.

\title{
Categories and Subject Descriptors
}

\author{
H.5.m Miscellaneous
}

\section{General Terms}

Measurement, Performance, Design, Reliability, Experimentation, Human Factors, Theory, Verification

\section{Keywords}

Permission to make digital or hard copies of all or part of this work for personal or classroom use is granted without fee provided that copies are not made or distributed for profit or commercial advantage and that copies bear this notice and the full citation on the first page. Copyrights for components of this work owned by others than the author(s) must be honored. Abstracting with credit is permitted. To copy otherwise, or republish, to post on servers or to redistribute to lists, requires prior specific permission and/or a fee. Request permissions from permissions@acm.org.

SAM'13, October 21, 2013, Barcelona, Spain.

Copyright is held by the owner/author(s). Publication rights licensed to ACM.

ACM 978-1-4503-2394-9/13/10...\$15.00.

http://dx.doi.org/10.1145/2509916.2509929

Mediated presence, mixed-reality, tangible interaction, user-centered design, quality of experience, shared media, social connectedness, spatial connectedness, information connectedness, real-time browser communication, COMPEIT

\section{INTRODUCTION}

The digital media industry is currently undergoing a profound transformation in which traditional distribution is supplanted by Internet-based distribution. Internet-based operators (e.g. NetFlix, HBO, Spotify), new actors, and production companies that favor for example YouTube or Vimeo as their preferred outlet increasingly challenge traditional TV and radio broadcast channels. An increasingly fierce competition can thus be foreseen between traditional media sectors and Internet based companies that operate in the digital media industry. The European Commission is also consulting widely on this matter, using the green paper "Preparing for a Fully Converged Audiovisual World: Growth, Creation and Values" [1].

On the other hand we also observe the progressive influence of modern technology on the personal and individual level within daily life. Media and communication technology started to change daily life when computer mediated communication technologies pushed work environments towards the public and private domain (e.g. public space, home and mobile environments) [2, 3, 4]. User generated content (e.g. blogs, review sites, online forms) and social media (e.g. Twitter, Facebook) applications are now mainstream as well as real-time communication applications such as Skype, Google Hangout or FaceTime, adding to the existing media ecology we have at our disposal today.

Many of today's communication means are however crude in their use and lack a real feeling of presence and immersion. Audio/video communication is still largely dominated by the 'talking heads paradigm', that is seeing each other's head in addition to voice communication. Furthermore, most Social Media (e.g. FaceBook, Pinterest) target the interaction belonging to 'weak ties', that is relationships characterized by less intimate and superficial contacts [5]. And, we are still mainly punching keys and clicking buttons instead of sharing life events and activities such as shuffling papers around the table during a work session, having diner together, playing poker together. Most of today's media and communication technology fails to address these forms of fluent, dynamic, and intimate interactions on a way we use these in real life.

New technologies and developments such as WebRTC, WebGL, real-time gesture and object recognition, 3D rendering and dirt cheap, credit-card-sized, fully functioning computer boards (e.g., Arduino, Raspberry Pi, Beaglebone) open a wealth of potential applications. The technology allows us to develop enhanced, more effective, dynamic, and natural ways of sharing information, doing things together, and socially connecting to one another in shared environments or spaces. Such new technologies are developed in the COMPEIT project, which is discussed subsequently.

\section{COMPEIT}

The COnnected Media and Presence from European Institute of Technology (COMPEIT) project, a new FP7 project starting in October 2013, addresses the changes foreseen above by offering innovative and competitive tools for the digital media industry with a main focus on an improved quality of experience in spatial, social, and information connectedness. COMPEIT builds on our current projects that have taken place in activities supported by the EIT-ICT Labs in the years 2011 and 2012. In the remainder of this paper we will briefly explain the plans for future research in COMPEIT.

COMPEIT technology is based on HTML5 for design, WebRTC for real-time "peer-to-peer" media distribution, and WebGL for 2D/3D rendering. It will first of all facilitate for example audience interaction and virtual eye contact. Second, COMPEIT explores the employment of tangibles for interaction and connected multiple devices for enhanced media experiences. Thirdly, COMPEIT takes advantage of 
mainstream ICT and Internet capabilities to render mixed-reality media, to create new attractive modes of advertising, and to remove dissonance between advertisers' need of viewer attention (hereby avoiding that viewers are 'drawn into' the advertisements or, de facto actively ignoring them).

Based on this, we have identified three research areas (see Figure 1), namely: 1) Spatial connectedness, 2) Social connectedness, and 3) Information connectedness. Spatial connectedness focuses on creating the illusion of a real space being extended beyond the screen in ways that allow us to actively participate on a shared stage and to interact with other more intimately (see Section 2.1). Social connectedness focuses on supporting and forming the explicit and tacit communication and belonging social dynamics among participants while performing shared activities or cooperative tasks in different settings and situations (see Section 2.2). Information connectedness focuses on providing the necessary information for maintaining effective interpersonal relations and awareness of others and their endeavors to enhancing collaborative activities (see Section 2.3).

Naturally these three research areas are overlapping in some aspects (see Figure 1), but taken together they provide insight in complete information, activity and communication spaces that should be applicable in many different domains and settings.

Some key issues being addressed in these research areas thus are:

- How to create illusions of extended space;

- How to enhance the support of explicit and tacit social dynamics belonging to different types of relationships, roles and communication and how to better support the information provisioning to create higher awareness of others and their endeavors, activities or processes in time to provide better cooperation.

We focus on these three research areas with the goal to enhance the quality of experience of systems, services and applications developed with insights from these areas. Quality of experience is defined as a construct consisting of many different possible experiences. Some experiences are closely related to life goals such as: relatedness, competence, security, control, even well being and the experience there-off in daily life, other are more or less related to periods of interaction (e.g. usability aspects such as efficiency and effectiveness or fun, enjoyment, engagement) and yet other experiences stem more or less directly from perception such as experienced presence, social presence, the illusion of extended space.

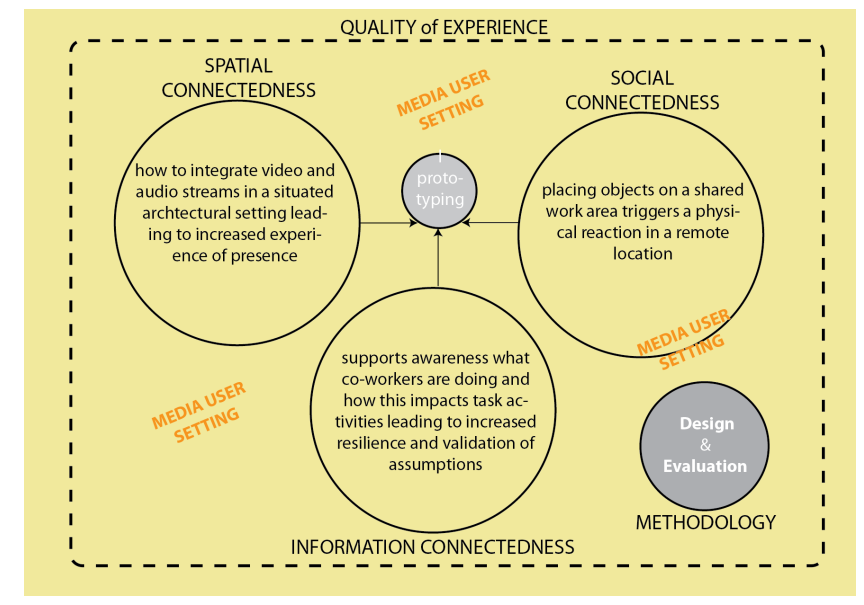

Figure 1 - the three research areas defined within the COMPEIT project

\subsection{Spatial Connectedness}

In shared mediated spaces, the individual experience of presence is negotiated as part of a process to establish trust and confirmation from the remote party/parties [2,3]. An on-going construction of a sense of place is informed by a series of 'reality tests' carried out by participants, who seek fast, consistent and reliable confirmation that the mediated architectural extensions can be trusted -in which case they can temporarily agree to suspend their judgment in favor of a 'willing suspension of disbelief'. The experience of presence is closely linked to spatial connectedness $[4,5]$. Ideally, we do not want to look at a video or a television screen, rather, we prefer the illusion that our real space is extended beyond the screen in ways that allow us to actively participate on a broadcasted stage, and to interact with others on e.g. a talk show.

The concept of spatial connectedness and presence design research allows us to investigate:

1) how video and audio streams that capture the face, body, and speech can be used more effectively to improve quality of media experiences through spatial presence, and

2) the spatial design considerations for embedding pictorial/ mediated spaces in physical space.

Video streams that come straight from a camera are strongly limited by the physical parameters of the cameras at both sides of the 
communication system. These limitations are associated with important problems such as the Mona Lisa effect. That is, if the second party is not looking straight into the camera because the camera is mounted above the screen, then the observer of the camera's image will have the impression that the person does not make eye contact. Using a camera in combination with even an inexpensive depth ranger (e.g. a XBox Kinect) would allow us to compute the image that the camera would have seen if it were not located above or next to the screen but at the position on the screen where the eyes of the second person are. This virtual camera would instantaneously solve the "Mona Lisa" problem.

Another important problem in viewing standard video displays is that the visibility of the display surface compromises the feeling of immersion, stereopsis, and the sense of realism of the 3D scene being viewed. Also this problem can be addressed by using virtual cameras because they can be used to create stereoscopic 3D content and viewpoint-yoked rendering. The ITEA2 VICOMO project has, for example, looked into video content analysis using multiple cameras and reasoned with context information, applied in scenarios for surveillance and digital signage.

The question what the design considerations are for integrating pictorial and physical spaces is important for creating the illusion that the picture plane is actually a window into the world behind. "Geometrically correct" is not necessarily the correct answer [7, 8]. In COMPEIT we look for what is psychologically correct. We do not only consider vision, but also spatial audio and touch.

Some examples of research questions that need to be addressed in field are:

- Does viewpoint-yoked rendering contribute to feelings of spatial presence? Does motion parallax provide a more reliable source of depth and distance information than binocular disparity?

- How critical is it for feelings of spatial presence that 3D sound matches the 3D vision?

- How does the light field in one location need to influence the light field in another location before it becomes truly realistic?

- What is the contribution from other spatial tools that must be monitored in processes where trust and consensus are established, hence with an impact on teamwork and its ethical considerations?

\subsection{Social Connectedness}

Much of today's user system interaction is artificial: we type, punch keys, and click buttons. Near future interaction with systems will be much more natural, based on the developments of touchscreens and interactive tables, movement recognition technologies (e.g. Xbox Kinect), tangible interfaces, speech recognition technology, and other types of input sensors. Much of today's human-to-human interaction is equally artificial and either synchronous (i.e. we chat or talk with and see each other at the same time), or it is asynchronous where we send emails, comment on status messages on social networks not necessarily on the same time. Today, when ending a synchronous interaction, we stop our current interaction and switch to doing something else. For professional situations this is often sufficient. However, in many other situations where deeper emotions are involved, e.g. with family members, there is a need for a better interaction paradigm.

In the social connectedness domain we need to investigate how to design mediated presence tuned to the current on-going social interaction, that is more distinct when people perform a shared activity or more non-distinct to indicate people want to interact with you, are just thinking about you or minding their own business but in a 'shared space' such as a living room. A simple example is a lamp that changes color based on remote interaction. Another example is a sick child that has a bracelet that can emit light and heat and when the parent touches her bracelet the child's bracelet would react. By the project start, the relevant technology will be getting to the point where the technology is not "in the way" and we can focus on how to use and apply it in a wider context instead. Mediated ambient presence can be divided into two groups, namely: "personal" and "room based". Personal is the type of devices that you carry with you on your person all the time, whereas room based devices are non-personal and available in the space around you. In the examples above, the lamp is room based whereas the bracelet is personal.

A common name for these connected devices for interacting is tangibles. The group of tangibles, e.g. advanced toys and home automation components that can be used for realizing the mediated ambient presence ambition, is growing rapidly. A key component is that these devices can be controlled wirelessly from a nearby computer or the device itself has full computer capabilities including wireless communications. A key success factor is that the tangibles can easily be deployed. That is, they are not special prototypes that cost very much to manufacture. Instead it is important to leverage on existing and coming technology that can be deployed without any large investments.

In the home automation area examples include wirelessly controlling electrical devices and cheap led lights can be remotely controlled to change color. In the area of toys, two relevant examples are Sifteo (www.sifteo.com) and Sphero (www.gosphero.com). The latter, Sphero, is a robotic ball that can move on its own, change color, detect if it is interacted with (e.g. picked up and shaken). The former, Sifteo, is a small cube with a touch sensitive color display and capabilities to detect if it is placed next to another Sifteo. Both these devices can communicate wirelessly with a nearby computer. Another interesting example is the Oriboo (http://www.oriboo.com/en/), which is also a small robotic ball that has "eyes" and audio output that can convey feelings in a way that is attractive to children. Yet another interesting technology that is available today is personal projectors that today are available in some mobile phones, where the phone becomes the interaction device and the visualization can be enlarged very easily. A research interest related to these kinds of technological developments is how to apply concepts such as these in a meaningful way in social interaction. How can we use technology like this to convey specific social meaningful information from parents to a child and the other way around? How can different (new) social meanings be attached to these technologies?

Using tangible devices we envision that they can also be used directly as part of the broadcast and thereby increase quality of experience by involving the user (within her social circle) in the activities of the actors in the broadcast. This all leads to a feeling of social connectedness between viewers and between viewers and participants in the broadcast and in turn this leads to a feeling of social connectedness. Many 
different aspects are important in maintaining social interaction and creating a feeling of social connectedness suitable to different contexts. Concepts for social interaction do apply to different target groups (e.g., elderly as well as children). The role of privacy, social norms/values, and laws are important aspects to take into account when designing the systems [9]. Information can be stored but communication sessions are often remembered differently from what was actually 'said'. Social connectedness is on one hand about a social feeling/affection needed and suitable for the contact and communication going on, and on the other hand it's about developing the right and suitable technology to support all the diverse aspects related to these feelings and affects. E.g. love buttons, hugging and glowing technology. Finally, what about measuring their effects? Do they truly result in the enhanced feelings and experiences for which they are developed?

Some of the research questions that will be addressed in COMPEIT are:

- Will the use of ambient mediated presence in different applications create an increased feeling of connectedness between patients and family (other people in their social circles) and in what specific way is social connectedness increased (e.g. support from the parents to child, pleasure and still being part of a social group by interacting with friends, etc.)?

- How can we create and support shared activities and important social dynamics using tangible devices and mediated presence technology necessary for experiencing social connectedness?

- Which type of tangible devices will give the best result for a better feeling of connectedness?

- How should tangible devices be deployed for the best result? Do users prefer personal or room based interaction? Or, a mix of both? Or are there specific aspects in social communication/interaction or shared activities that are typically room based or personal based?

- How should the software behind the tangibles be designed to allow for scalability and easy deployment?

- Which technologies, e.g. Kinect, reality based interaction (e.g. ambient technology, audio/video communication, social media) can we identify as affecting presence and awareness and through that social connectedness?

- What social interactions are supported and how do they affect a feeling of social connectedness (playing a game, working together, giving a hug, learning together, eating together)?

\subsection{Information Connectedness}

Collaboration is affected negatively when actors are distributed geographically or when actors are separated in time [10] because actors experience complications in the development of effective interpersonal relations, experience more frequent communication mishaps, and have a lower awareness of team members' endeavors [10]. In addition, the common denominator in distributed settings is the failure to sense states, observe actions directly, and witness responses of remote actors [11], which increases awareness what your co-workers are doing and how this impacts the collaborative activities. The omission of this information disrupts anticipation of information needs, reduces resilience, and hardens validation of assumptions.

Observing actions of remote collaborators helps to anticipate the next step in the collaborative activities [12], without requiring explicit communicative acts to coordinate activities. Moreover, the observations allows to sense whether the co-actor is coping adequately or requires assistance, leading to a group that is highly flexible and resilient in a variety of unexpected situations. In addition, information that shows that assumptions are incorrect is important in remote collaborations because actors make assumptions to compensate when limited information is available. A classic case where incorrect assumptions seriously worsened the situation relates to the Mont Blanc tunnel fire in 1999 [14].

Real-time mediated presence systems provide a rich image of the remote actor, potentially showing actions, states, and responses. However, these systems are typically limited to the time frame of the call. Activities executed outside the time frame of the call may have an impact on the shared activity. The time between call exceeds in many collaborative settings the duration of the call, limiting the opportunity to synchronize activities. Being aware of such activities is thus important in order to synchronize joint activity. This kind of information proves valuable in dynamic settings, such as a hospital, as it allows validating assumptions and being aware on activities impacting the joint activity.

Information Connectedness refers to the information that is required to feel connected and collaborate effectively while being distributed. Likewise, viewing an interactive broadcast together is a shared collaborative activity. Information connectedness would allow non-linear play-out of the broadcast based on the collaborative viewing experience. Recently, much progress has been made in the area of automatic recognition of emotions and intentions (for example in the ITEA2 "Empathic Products" project) and data mining the social networks for trending topics (for example in the FP7 "SocialSensor" project). In the Information Connectedness domain we now need to investigate how to design for awareness related to joint activities regarding mediated presence systems. In particular, in this domain it is explored:

- In which way an intelligent software agent needs to be designed so that actors are increasingly aware of elements that affect the shared activity.

- What the effects of such a software agent would be on collaboration, resilience, and adaptation.

- How information must be presented to the actors to allow them to interact naturally and to optimize situation awareness.

COMPEIT therefore aims to develop an Information Connectedness Agent that puts forward relevant information regarding the shared activity. Such information should put the information in the periphery of the process, allowing to browse this information at convenient times increasing the chance to observe interesting information regarding the shared activity with minimal interruption. The agent aids the human to observe what remote actors are doing adding valuable information about the context in which work is achieved. 


\section{COMPEIT innovations and user involvement}

COMPEIT will develop and validate three key services:

1. Shared Experience with Tangible Interaction (SETI) is an Internet-based service, which allows an audience to experience enhanced live media together, complemented by tangible and interactive games. Distributed media is enhanced by the addition of virtual eye contact to increase the experience of witnessed presence. Scalability for larger audiences is granted through media distribution network support and audience partitioning into workable groups of people, based on social networks or user profiling.

2. Broadcast Presence Studio (BPS) is a web-based environment for mixing live (or stored) media with other types of web-based media, such as photos, maps, web pages or even full web applications. Further, this will facilitate pre-processing for virtual eye contact, using multiple cameras or full stereoscopic field cameras. The BPS will also facilitate publishing through the SETI service.

3. Mixed-Reality Interaction is an advanced HTML5/WebRTC-based service where content generated by the BPS service can be mixed into the user's physical environment by means of ambient or wearable 3D devices. This service will be experimental, aiming primarily to explore its use for social entertainment and interaction, as well as for mixed-reality advertisements in view of new revenue streams for the digital media industry.

These three key services will be bundled in to several main prototypes. COMPEIT technologies will immediately be implemented in a "release-early, release-often" fashion. User validation of COMPEIT's key service will be done at two test sites, namely the Princess Maxima Center for Child Oncology in Utrecht (the Netherlands) and at Karolinska University hospital in Stockholm, Sweden. At these test sites we evaluate the services in mixed personal/work settings. These pilots will focus mostly on face-to-face and narrow casting communication services.

We will systematically analyze users' needs and the impact of COMPEIT technology on ethical and traditional values from a holistic quality of experience perspective. Especially, ethical values (e.g. privacy, data ownership, accountability) are coming under pressure in new media technologies. In addition to the focus on ethical values, classical values (e.g., performance, efficacy, team coherence) are also included in the analyses.

Users' needs values are dependent on the application area. This means that the user must be investigated in the context of the application area and the envisioned technology. Consequently, the interplay between users, the application area and the envisioned technology have to be analyzed in order to be able to improve the user's quality of experience optimally. That is, do a deep domain analysis of media and presence in the context of the user. The analyses will lead to a set of requirements, claims, use cases and application scenarios that will later on be improved iteratively via stakeholders' reviews using agile software development methods and the application of situated Cognitive Engineering methodology. Claims describe the a-priori expected effect related to each requirement. A claim can describe a positive expected effect (e.g. a performance increase) or a negative expected impact (e.g. display clutter). Each claim, use case and scenario is linked explicitly to a requirement.

In COMPEIT we combine Situated Cognitive Engineering and Value Sensitive Design into a user-centric iterative design methodology [9]. This methodology involves end-users and other stakeholders in the continuous iteration of analyses, development and innovation. Whereas Value Sensitive Design refers to an approach that accounts for human values in a principled and systematic manner throughout the design process, Situated Cognitive Engineering systematically studies the domain, its underlying cognitive theories and relevant technologies in order to define value sensitive requirements that is contextualized with application scenarios and justified by claims regarding their effect on operational use.

Innovative media companies in Sweden, the Netherlands and in Israel/UK/Europe will be invited to pilot the use of the SETI, BPS and mixed reality services, for example for politics and sports productions. The above services will be evaluated iteratively in online Experience Lab sites. These evaluations mostly target media broadcasting. Specific attention will go out to user interactivity and non-linear play-out of the broadcast.

\section{CONCLUSIONS \& DISCUSSION}

Society currently faces increasingly complex challenges and we need to facilitate sustainable patterns of behavior, accepting that the boundaries between the natural and the technological have been repeatedly called into question. In our society of ubiquitous computing, technology has encroached into how we 'enact being alive'. What are the unstated boundaries of one's profession? It suffices to agree with Herbert Simon's claim that we live in a world of artificial artifacts designed by humans [15]; with Donna Haraway's argument that new technologies have become inseparable from our bodily identities [16]; or with Manuel Castells that social change requires the ability to shape the human mind, the most fundamental form of power [17] to realize that disciplinary boundaries are due to be dissolved. Viable communication solutions require creative and innovative thinking on a number of levels, not least ethical concerns for our new 'cyborg identity'. How might we navigate between our natural and a range of mediated presence(s), as currently designed by presence technologies, transaction technologies, social network technologies, surveillance and identification technologies, etcetera?

In the current paper we have given an overview of the mediated presence landscape and have outlined our approach to improving quality of experience in connected media en mediated presence systems. Specifically we aim to improve three forms of connectedness, namely spatial, social and informational. We aim to develop modular mainstream systems in the form a SETI, BPS and Mixed reality components and demonstrate their potential by building main prototypes for mixed personal and professional communication systems in two hospital settings. Furthermore we demonstrate their applicability for media broadcasting of new media with deep feelings of immersion, connectedness and presence. We have an interesting time ahead of us!

\section{ACKNOWLEDGEMENTS}


We are grateful for the support we received through the Mediated Presence activity that is sponsored by the EIT-ICT Labs. The EIT-ICT Labs is a knowledge and innovation community setup by the European Union. Its mission is to drive leadership in innovation in ICT for economic growth and quality of life. In addition, we express our thanks to all other partners in the COMPEIT core team: Johan Bengtsson, Leif Handberg, and Seppo Valli. The current paper is based to a large extent on the writing we have done collaboratively as team over the last few months of 2012. Our ongoing activities will be documented shortly at http://www.compeit.eu.

\section{REFERENCES}

[1] European Commision (2013). Preparing for a Fully Converged Audiovisual World: Growth, Creation and Values

[2] IJsselsteijn, W.A. (2004). Presence in depth. Doctoral dissertation. Eindhoven: Eindhoven University of Technology

[3] IJsselsteijn, W.A., \& Riva, G. (2003). Being there: The experience of presence in mediated environments', In: G. Riva, F. Davide, \& W.A. IJsselsteijn (Eds.) Being there: Concepts, effects and measurement of user presence in synthetic environments. Amsterdam: IOS Press

[4] Buxton, W. (1992). 'Telepresence: Integrating Shared Task and Person Spaces', Proceedings of Graphics Interface '92, pp. 123-129

[5] Gullström, C. (2010). Presence design: Mediated spaces extending architecture. Doctoral thesis. Stockholm: KTH.

[7] Ling, Y., Nefs, H.T., Brinkman, W.P., Qu, C. \& Heynderickx, I. (2012). The effect of perspective on presence in the virtual environment for different display devices. In revision

[8] Vishwanath, D., Girshick, A.R. and Banks, M.S. (2005) Why pictures look right when viewed from the wrong place. Nature Neuroscience, 8(10), 1401-1410

[9] de Greef, T.E., Mohabir, A; van der Poel, I. and Neerincx, M. sCEthics: Embedding Ethical Values in Cognitive Engineering, paper presented at the European Conference of Cognitive Ergonomics, ACM.

[10] Powell, A., Piccoli, G., \& Ives, B. (2004). Virtual teams: A review of current literature and directions for future research. The DATABASE for advances in information systems, 35(1), 6-36

[11] Thompson, L. F., \& Coovert, M. D. (2006). Understanding and developing virtual computer-supported cooperative work teams. In: C. Bowers, E. Salas, \& F. Jentsch (Eds.), Creating high-tech teams: Practical guidance on work performance and technology. Washington, DC: American Psychological Association

[12] de Greef. T.J. (2012). ePartners for dynamic task allocation and coordination. Doctoral dissertation. Delft, the Netherlands: Delft University of Technology

[13] Heath, C., \& Luff, P. (1992b). Media space and communicative asymmetries: Preliminary observations of video-mediated interaction. Human-Computer Interaction, 7(3), 315-346

[14] Minister of the Interior - Ministry of Equipment, Transportation and Housing (1999). Task Force for Technical Investigation of The 24 March 1999 Fire in The Mont Blanc Vehicular Tunnel - Report of 30 June 1999 (English translation).

[15] Simon, H. A. 1969. The Sciences of the Artificial (Cambridge: MIT Press)

[16] Haraway, D. 1991. Simians, Cyborgs, and Women: The Reinvention of Nature (New York: Routledge)

[17] Castell s, M. 2009. Communication Power (Oxford: Oxford Univer sity Press) 